\title{
Mobility Metrics Based Classification \& Analysis of Mobility Model for Tactical Network
}

\author{
Santosh Kumar, S. C. Sharma, Bhupendra Suman \\ Indian Institute of Technology, Roorkee \\ \{amu.santosh@gmail.com, subhash1960@rediffmail.com, \\ bhupendra_sumanerediffmail.com\}
}

\begin{abstract}
In the tactical network scenario mobile nodes are require to attain high degree of mobility without the aid of prior network infrastructure. To access the efficient and reliable communication it must be based on tactic scenario like specified area, task specified unit etc. in real time. The performance of this system in real time is very difficult to do because of it need field test, several hundred devices as well man-power. The evaluation of algorithm and protocol simulation is another alternative to evaluate the performance of tactical network. The simulative performance evaluation relies on models used in the network. This paper basically provides survey and categorization of the mobility model on account of mobility metrics, as well as analysis of those models which meet the tactical scenario requirement tabulated in table 2 .
\end{abstract}

Keywords: Tactical communication network, Mobility metrics, mobility model, tactical scenario

\section{Introduction}

Tactical mobile ad hoc network (MANET) is a collection of mobile nodes forming a temporary network, without the aid of pre-establishment network infrastructure. Commercial wireless technologies are generally based on towers and high-power base stations. They are fixed in location and relationship to their client devices. These are not exact option to fulfil the requirement of Defence. It need technology base for future military network. Sudden catastrophe (Calamity) may be natural (e.g. flood, tornado , volcano eruption, earthquake, or landslide etc.) and man-made ( e.g. explosion, fire, etc. ) need very high rescue operation with reliable communication among units working these disaster areas. To access the efficient and reliable communication it must be based on tactic scenario like specified area, task specified unit etc. in real time. The performance of this system in real time is very difficult to do because of it need field test, several hundred devices as well man-power. The evaluation of algorithm and protocol simulation is another alternative to evaluate the performance of tactical network. The simulative performance evaluation relies on models used in the network.

In the literature there are already review has been done on mobility models [1, 2, 3]. This paper is exploring mobility models and which meets for performance evaluation of tactical network.

The rest of the paper organize as follows: Section 2-describes about tactical communication network, Military tactic and battle space and section 3 describes the classification of mobility model and its performance based on mobility metrics to meet the tactical scenario requirements (sub-section $3.1-3.5)$

\section{Tactical Communications Networks}

Tactical communications networks use wireless technology to achieve a high degree of mobility. Unlike cellular networks where centralized infrastructures (base stations) are static. Tactical communications networks cannot rely on static infrastructure for following reasons.

1. Movements are driven by tactical reasons

DOI : 10.5121/ijngn.2010.2305 
2. Unit needs optimal path to destination

3. Destination depends on working site based on tactical issues

4. Work force specially distributed and mobile.

Units may be geographically dispersed, command and control is usually preferred to be centralized. This centralization may be to one or to a hierarchy of points or could be an algorithm based dynamically selected.

Military tactics are the collective name for methods used by units up to a division in strength in engaging and defeating an enemy in combat. Changes in philosophy and technology over time have been reflected in changes to military tactics. Tactics should be distinguished from military strategy, which is concerned with the overall means and plan for achieving a long-term outcome, and operational art, an intermediate level in which the aim is to convert the strategy into tactics.

Battlespace is a significance of unified military strategy to integrate and combine armed forces for the military theatre of operations, including air, information, land, sea and space to achieve military goals in the distributed form with allocation of specific task to a unit . In conclusion with respect to battlespace analysis yields the important necessities as:

1. Tactical areas

2. Obstacles

3. Optimal path

4. Unit/Group moment with respect to a mobility model

5. Heterogeneous velocity

\section{Classification of mobility metrics}

The mobility model can be classified with different kind of mobility metrics classes like randomness, dependencies and restrictions that are considered in table by several studies in the literature [4].

Table1. Classification of mobility metrics \& its characteristics

\begin{tabular}{|l|l|l|}
\hline Sl.No & Metrics & Characteristics \\
\hline 1. & Random based & Without any dependencies and restriction invoked in model \\
\hline 2. & Temporal dependencies & A node actual movement influenced with its past movement \\
\hline 3. & Spatial dependencies & $\begin{array}{l}\text { The movement of a node influenced by node around it } \\
\text { happen in group mobility. }\end{array}$ \\
\hline 4. & $\begin{array}{l}\text { Geographical } \\
\text { restrictions }\end{array}$ & Node movement restricted in certain geographical area \\
\hline 5. & Hybrid structure & $\begin{array}{l}\text { All mobility metrics classes are integrated to attain the } \\
\text { structure }\end{array}$ \\
\hline
\end{tabular}

\subsection{Random movement based model}

The random way point mobility model was first introduced by Broch et al [7]. it is very popular model in modern research and can be considered as a foundation of building other mobility model. It includes pause times between changes in direction and speed [8].A mobile node stay in one location for certain period of pause time. In original model mobile node are distributed randomly in the simulation area between speed [Vmin, Vmax]. After reaching at waypoint, the node wait again a constant pause time and do it again choose next waypoint. This process repeated after choosing pause time from interval [Pmin, Pmax] [8]. In [9] it is reported that the average velocity of node is decreasing during simulation if $\mathrm{Vmin}=0$. Therefore $\mathrm{Vmin}>0$ and Pmax $<\infty$ should be chosen. In several publication, it has been reported that the nodes cluster in the middle of the simulation area $[4,10,11,12]$ as shown in following figure. 1 


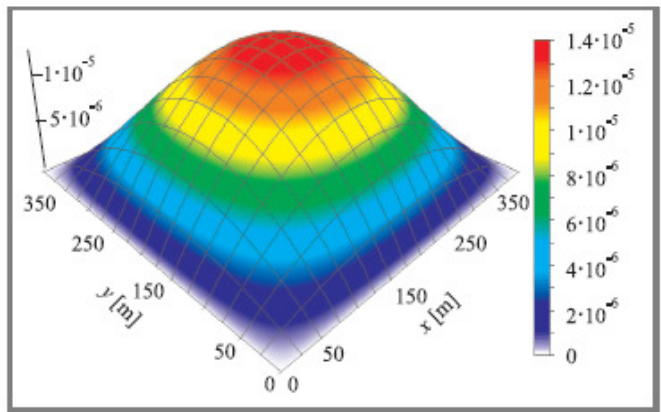

Figure 1. Nodes density for Random Way Point Model

So distribution and movement of the node across the simulation area does not fit to the characteristics of realistic movements.

\section{Modified random waypoint model}

In this model, the chosen destination points can only be located at the borders of the system area. For example [12], the destination points in a circular area are taken from

$$
f\left(r_{d}, \theta_{d}\right)=\left\{\begin{array}{lc}
\frac{1}{2 \pi r_{m}} & \text { for } \quad r_{d}=r_{m} 0 \leq \theta_{d}<2 \pi \\
0 & \text { else }
\end{array}\right.
$$

where simulation area of radius $\boldsymbol{r}_{m}=\boldsymbol{r}_{d}=\sqrt{x_{d}^{2}+y_{d}^{2}}$ and $\theta_{d}=\arctan \left(\frac{y_{L}}{x_{d}}\right)$

It is also known as Random Border Point Model (RBPM). In this model nodes are located at the border and try to reach from source to destination along with same border with high probability.

\section{Random direction mobility model}

The random direction mobility model [E.M.Royer et al-13] introduced to overcome the density of node cluster in the centre part of simulated area of Random way point mobility model. In this model node choose random direction between 0 and 359 degrees and travel border of the simulation area from the centre and once node meet to boundary, pauses for a specified time then it further chooses angular direction between 0 and 180 degrees and repeat the process.

Modified random direction model: In this model, nodes select a direction degree as before, but they may choose their destination anywhere along that direction of travel. They do not need to travel all the way to the boundary [13].

\section{Clustered mobility model}

In this mobility model all nodes of the network classified into cluster head node and cluster member node. Cluster member node is one hop away from the cluster head. Cluster head maintain the IP address of all its cluster member and cluster members also records the IP address of its Cluster head. An adaptive mobile cluster algorithm can sustain the mobility perfectly and maintains the stability and robustness of hierarchical network architecture [14]. In a conventional clustering ad hoc network, the mobile nodes are separated into groups called clusters. A cluster of the ad-hoc network, generally consists of three types of nodes: cluster heads $(\mathrm{CHs})$, gateway nodes and normal nodes (cluster member node) [15]. 


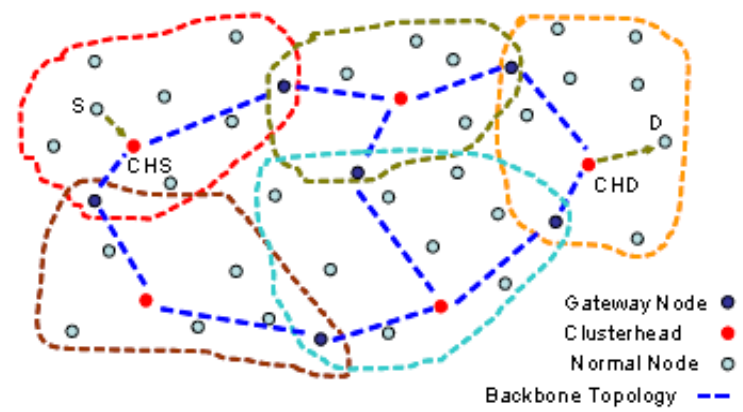

Figure 2. Cluster structure for MANET

\subsection{Temporal Dependencies}

In this mobility model, a node actual movement influenced with its past movement.

\section{Gauss-Markov model}

This model was designed initially for the simulation of a PCS [16], to attain different level of randomness with tuning parameter $\propto(0 \leq \alpha \leq 1)$. The value of speed and direction at the $\mathrm{n}^{\text {th }}$ interval is based upon the value of speed and direction at the $(n-1)^{\text {th }}$ interval and a random variable using the following formulas.

$$
\begin{aligned}
& s_{n}=\propto s_{n-1}+(1-\propto) \bar{s}+\sqrt{\left(1-\alpha^{2}\right) s_{n-1}} \\
& d_{n}=\propto d_{n-1}+(1-\propto) \bar{d}+\sqrt{\left(1-\alpha^{2}\right) d_{n_{n-1}}}
\end{aligned}
$$

where $s_{n}$ and $d_{n}$ are the new speed and direction of the mobile node at interval $\mathrm{n}$.

at each interval the next location is based on the current location, speed and direction of movement. The total random values (or Brownian motion) could be obtained if $\propto=0$ and linear values could be obtained by setting $\propto=1$ [16]. The time interval $n$, a mobile node position is given by the formulas:

$$
\begin{aligned}
& x_{n}=x_{n-1}+s_{n-1} \cos _{n-1} \\
& y_{n}=y_{n-1}+s_{n-1} \operatorname{sind}_{n-1}
\end{aligned}
$$

where $\left(x_{n} y_{n}\right)$ and $\left(x_{n-1}, y_{n-1}\right)$ are the $\mathrm{x}$ and $\mathrm{y}$ coordinate of mobile node position at the $\mathrm{n}^{\text {th }}$ and $(\mathrm{n}-1)^{\text {th }}$ time interval.

\section{Smooth Random Model}

The smooth random model primarily proposed by Bettstetter $[17,18]$. This model depicts the movements of node more smooth and realistic; it eliminates sharp turns and sudden stops. In this model, a preferred set of speed is defined with a high probability which is allocated to each of them, instead of uniform distribution of speeds between $\left(0, \mathrm{~V}_{\max }\right)[2]$.

$$
\mathrm{P}(\mathrm{V})=\left\{\begin{array}{c}
P(V=0) \delta(V) \\
P\left(V=1 / 2 V_{\max }\right) \delta\left(V-1 / 2 V_{\max }\right) \\
P\left(V=V_{\max }\right) \delta\left(P-V_{\max }\right) \\
\frac{1-N(v-0)-\Gamma\left(v-1 / 2 v_{\text {muls }}\right)-\Gamma\left(V-v_{\text {mux }}\right)}{V_{\max }} \\
0
\end{array}\right.
$$

$$
\begin{gathered}
V=0 \\
V=1 / 2 V_{\max } \\
V=V_{\max } \\
0<V_{\max }<1
\end{gathered}
$$


When a change direction occurs, a new movement direction is selected randomly from $(0,2 \pi)$. If the $\varphi^{*}\left(\mathrm{t}^{*}\right)$ is the new direction at time $\mathrm{t}^{*}$ and $\varphi\left(\mathrm{t}^{*}\right)$ is the old direction then the direction difference will be $\left[\Delta \varphi\left(t^{*}\right)\right]=\left[\varphi^{*}\left(\mathrm{t}^{*}\right)-\varphi\left(\mathrm{t}^{*}\right)\right]$ therefore

$$
\Delta \varphi\left(t^{*}\right)=\left\{\begin{array}{cc}
\varphi^{*}\left(t^{*}\right)-\varphi\left(t^{*}\right)+2 \pi & -7 \pi<\varphi^{*}\left(t^{*}\right)-\varphi\left(t^{*}\right)<-\pi \\
\varphi^{*}\left(t^{*}\right)-\varphi\left(t^{*}\right) & -\pi<\varphi^{*}\left(\mathrm{t}^{*}\right)-\varphi\left(\mathrm{t}^{*}\right) \leq-\pi \\
\varphi^{*}\left(t^{*}\right)-\varphi\left(t^{*}\right)-2 \pi & \pi<\varphi^{*}\left(\mathrm{t}^{*}\right)-\varphi\left(\mathrm{t}^{*}\right) \leq 2 \pi
\end{array}\right.
$$

It does not focus on the regular movement of the nodes and do not considers obstacles so that it violets the realization of tactical scenarios.

\subsection{Spatial Dependencies}

In spatial dependency nodes movements are in group fashion. The movement of a node influenced with other nodes around it.

\section{Reference-Point Group Mobility Model}

It is one model which realizes the spatial dependence with the use of reference points. The RPGM model enables the random motion of the group/cluster and also enables the individual motion of a node in its own group/cluster [19]. Every group/cluster has a logical centre. The mobility characteristics like motion behaviour, location, speed, direction etc .of the entire group/cluster depends over the logical centre motion. All nodes are distributed within the geographical scope of the group.

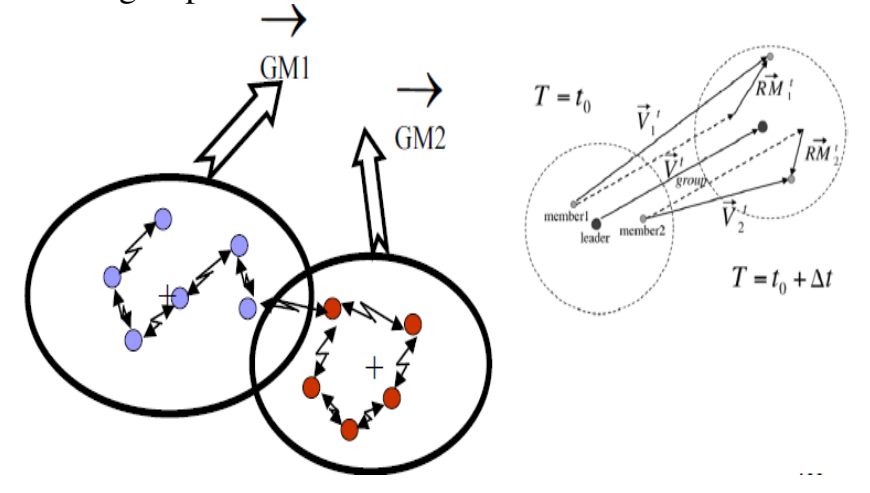

Figure 3. Group mobility model

This model realizes the spatial dependency of each node of a group with logical centre. It is suitable for tactical network scenario to shoot out tactical requirements.

\section{Structured- group- mobility model}

In this mobility model nothing like random movement vector [20]. The nodes of a group movement are fixed and non changing formation. The movement of a groups are indicated for common goal, geographic etc. examples of this model like fire-fighter in a building, crowed control action by police officers, disaster rescue operation by military, military vehicles on the move etc. are significance of a structure on the group. In spite of its more complex in nature than other previous mobility model, it signifies the advantages of real-world behaviour of groups with inherent structure. This model is useful for fire- fighting operation in a building and military scenario in battlefield. 
In the literature survey there are several variance of the RPGM model e.g pursue model, column model, nomadic community model [1].

\section{Community based mobility model}

In mobile ad hoc network mobile devices are carried with the help of humans so the movement of these devices are based on human decision and socialization behaviour [21] .For this it is important to model the behaviour of individual moving group and between groups, as clustering is also typical ad hoc networking deployment scenarios of disaster relief teams, platoons of soldiers, groups of vehicles, etc. For capturing this behaviour it require community based mobility model and heavily dependent the structure of the relationships among the people carrying the devices. This model supports the optical path requirements for source to destination node and modification could be carried out having knowledge of group mobility for tactical network scenario too.

\subsection{Geographical restrictions}

In the graph-based mobility model vertices are the possible destination node and the edges are possible route. It has very different approaches to restrict the nodes movement in certain part of the simulation area [4].

- Graph-based approach

- Geographic division based- approach

- Map-based approach

- Voronoi-based approach

\section{Graph-based approach}

It is possible to simulate Random mobility model for conventional scenario in MANET possessing certain area randomly .But, in real world scenario random mobility is always not possible such as the human mobility as well as vehicle mobility could not be possible randomly. So for realization of this fact graph based approach play very significant role [23]. In this scenario real entity works as source node and destination node. The connecting path is known as edge. In this approach random movement of real entity could be possible having absolute shortest path and velocity. It is not quite enough approach for tactical networks requirements.

\section{Geographic division based-approach}

\section{Area-graph-based mobility model}

This model tries to realize division of geographical area in the form of cluster (sub-area) with higher node density and paths in between lower node density [23]. The cluster is recognize as a vertices of the area graph while path as edges. The movement of cluster node could be managed with random way point model. Similar approach is used in CosMos [24].

These models are suitable to realize tactical networks requirements.

\section{Map-based mobility model}

Map-based mobility model comprises to restrict the movement area geographically. It could be useful in modelling movement in an urban area. The scenario is composed of a number of horizontal and vertical streets. The nodes movement are along the horizontal and vertical streets on the map with probability factor of node turn at intersection point of streets. It imposes too 
geographical restriction on node mobility. Under the UMTS standardization, Manhattan-grid model is specified for this approach[24].

As per concern of tactical scenario requirements it is not appropriate for tactical networks.

\section{Voronoi-based approach}

The Voronoi-based approach was initially introduced in obstacle mobility model $[25,26]$ to determine the movement path or area using Voronoi digram. In this model edges of the building results used as input to generate the Voronoi digram [27]. The movement of graph consists Voronoi digram and vertices (buildings) and realized analogous to graph based model. It does not provide optimal path, so it can not meet the requirement of tactical networks.

\subsection{Hybrid structure}

All mobility metrics classes are integrated to attain the structure like-relative speed, spatial dependence, temporal dependence and node degree/ clustering. All the metrics of table 1, could be integrated to obtain the hybrid structured mobility model as in below figure 4 .

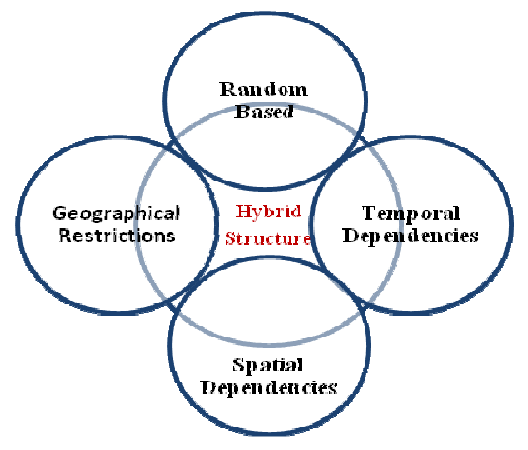

Figure 4. Hybrid structure mobility model

There are some models which meet the requirements of the hybrid structure.

- Complex vehicular traffic models

- User-Oriented mobility data-model

- Tactical scenario Model

- Disaster-area model

\section{Complex vehicular traffic models}

Vehicular traffic mobility is an extensive research based on vehicles movement along with urban streets which itself establishes networks. Vehicles are considered as mobile nodes along with pathway. Having its complex traffic behaviour there are several mobility model introduced by considering certain traffic movement rules like topological map, vehicle speed, obstacles, desirable points etc. Free way mobility model, Mahattan Mobility model [28], are the few model which are introduced for vehicular traffic.

\section{Free way mobility model}

This mobility model includes the spatial, high temporal dependencies and also imposes strict geographical restriction [6]. The mobile node velocity is temporally dependent on its previous velocity and also the velocity of a mobile node is also influenced with other mobile node 
moving in the same lane inside certain radius (spatial dependence). All mobile nodes movement are imposed strict geographical restriction.

\section{Manhattan Mobility model}

It predicts mobility pattern of mobile nodes on bidirectional street and useful in modeling of urban area vehicular traffic [29]. It is composed of horizontal and vertical streets map which causes geographical restriction of networks. The model realizes spatial and high temporal dependencies.

The above both models are useful for vehicular traffic modeling but inefficient for all aspects of tactical scenario.

\section{User-Oriented mobility data-model}

Mobility scenario is a significant asset which plays a vital role for performance evaluation of mobile networks. Illya Stepanov et.al[30] introduces an user-oriented mobility model data model which provide an modeling approach for complex mobility scenario. It includes three components-

1. Consideration of spatial environment (Roads, streets etc ), restriction of mobile users, as well as attraction points (Spatial model).

2. Activity sequence made by users as per their observation e.g. sequence of attraction points(User trip model)

3. Mobility model must reflects the dynamics of the mobile user (Movement dynamic model) introduce temporal and spatial dependencies.

This model could be used for modeling of typical movements of mobile nodes in daily routine basis ( cf. [31]).

Some of the tactical requirements could be realized using this model however tangible realizations of the requirements are not specified particularly in this model.

\section{Tactical scenario Model}

There is some realistic scenario which considered less artificial approach instead of random movement for analysis of routing protocol performance [Per Johansson et al 32]. In this realistic scenario, catastrophe scenario is also there which focuses the relatively slow and fast movement of the nodes. It could be considered for obstacles, group movements and tactical scenario. There are others scenario models are reported for platoon movement in city area [Steffen Reidt et al 33].

\section{Disaster-area model}

This could be get in details in a model [Nils Aschenbruck et al 5] which represents a realistic movements based over disaster area scenario. It focuses heterogeneous area-based movement on optimal path avoiding obstacles joining or leaving of nodes as well as optional group mobility. To realize the area-based movement, the simulation area is divided into tactical sub area. The area is classified civil-protection concept as in figure 6. 


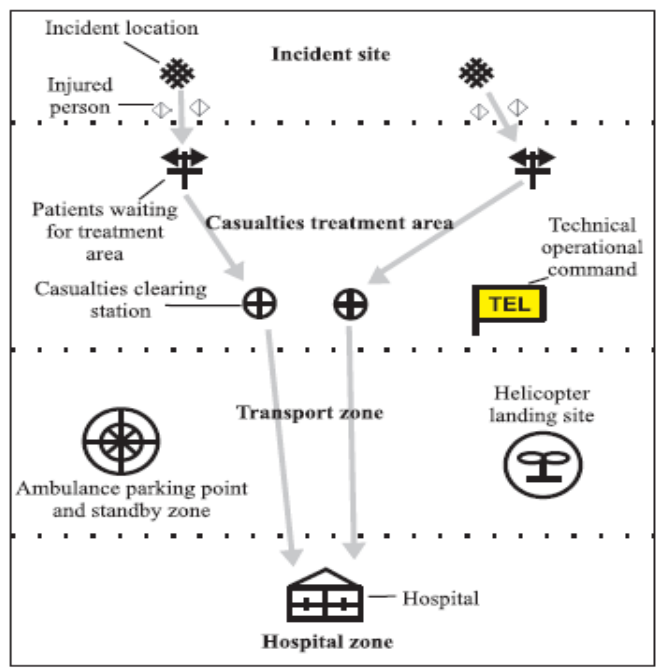

Figure 5. Separation of Room method in civil protection

It shows different tactical area and its tactical facilities like-

- Incident site- place where calamity actually happen, in this area affected and injured people as well as facilities are found and calamity could be minimized.

- Causalities treatment area- it divided into two part and closer to incident site.

First- patient waiting for treatment area- usually closer to incident site and provide rescue operation and treatment.

Second-causalities clearing area-provide transportation to affected people

- Technical operational command-usually located in causality treatments

- Transport Zone - it provide transportation service to hospital

- Hospital zone- it provide treatment facilities to affected people

This analysis carried out that each node is assigned to one of these tactical areas. In some tactical area both stationary and mobile nodes (transport) act its specified task which movements are based on random mobility model. It has heterogeneous speeds for different tactical area and its operation. It also uses graph based approach to get optimal shortest paths avoiding with obstacles between tactical areas. Group mobility is realized as optional characteristics for disaster areas, as in civil protection there may only be one reference point for a group, so it is realized similar to RPGM [19].The movement of each node in a group is calculated with respect to reference point. 
International Journal of Next-Generation Networks (IJNGN) Vol.2, No.3, September 2010

Table 2. Mobility model which meets the tactical scenario requirements

\begin{tabular}{|c|c|c|c|c|c|c|c|}
\hline \multirow{2}{*}{\multicolumn{2}{|c|}{ Mobility Model with Metrics }} & \multicolumn{6}{|c|}{ Tactical scenario requirements } \\
\hline & & \multirow[t]{2}{*}{ 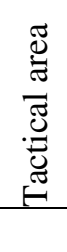 } & \multirow[t]{2}{*}{$\begin{array}{l}\frac{\tilde{U}}{\tilde{U}} \\
\tilde{\tilde{U}} \\
\tilde{0}\end{array}$} & \multirow[t]{2}{*}{ 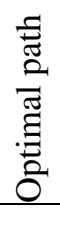 } & \multirow[t]{2}{*}{ 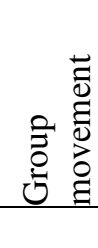 } & \multirow[t]{2}{*}{ 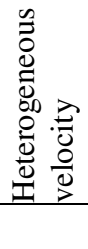 } & \multirow[t]{2}{*}{ 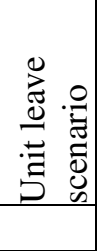 } \\
\hline \multicolumn{2}{|l|}{ 1. Random movement based model } & & & & & & \\
\hline - Random-waypoint & [7] & $\mathrm{E}$ & & $\mathrm{Y}$ & & $\mathrm{E}$ & \\
\hline - Modified Random Waypoint & {$[12]$} & $\mathrm{E}$ & & $\mathrm{Y}$ & & $\mathrm{E}$ & \\
\hline - Random direction mobility & {$[13]$} & $\mathrm{E}$ & & $\mathrm{Y}$ & & $\mathrm{E}$ & \\
\hline - Modified random direction & {$[13]$} & $\mathrm{E}$ & & & & $\mathrm{E}$ & \\
\hline - Clustered mobility & {$[14,15]$} & $\mathrm{E}$ & & $\mathrm{Y}$ & & $\mathrm{E}$ & \\
\hline \multicolumn{8}{|c|}{$\begin{array}{l}\text { 2. Temporary Dependencies based } \\
\text { model }\end{array}$} \\
\hline - Gauss-Markov & {$[16]$} & $\mathrm{E}$ & & & & $\mathrm{E}$ & \\
\hline - Smooth random & $\begin{array}{l}{[2,17,} \\
18]\end{array}$ & $\mathrm{E}$ & & $\mathrm{Y}$ & & $\bar{Y}$ & \\
\hline \multicolumn{8}{|c|}{ 3. Spatial dependencies based model } \\
\hline - Reference point group & {$[19]$} & $\mathrm{E}$ & $\mathrm{E}$ & E & $\mathrm{Y}$ & $\mathrm{E}$ & $\mathrm{E}$ \\
\hline - Structured- Group & {$[20]$} & $\mathrm{E}$ & & $\mathrm{Y}$ & $\mathrm{Y}$ & E & \\
\hline - Community based & {$[21]$} & $\mathrm{E}$ & & $\mathrm{Y}$ & $\mathrm{Y}$ & $\mathrm{E}$ & \\
\hline \multicolumn{8}{|l|}{ 4. Geographical Restrictions } \\
\hline - Graph-based & {$[23]$} & & $\mathrm{E}$ & $\mathrm{Y}$ & & $\mathrm{E}$ & \\
\hline - Geographic division based & {$[23]$} & $\mathrm{Y}$ & $\mathrm{E}$ & $\mathrm{E}$ & & $\mathrm{E}$ & \\
\hline - Map-based & {$[24]$} & & & & & $\mathrm{E}$ & \\
\hline - Obstacle based & {$[25,26]$} & & $\mathrm{Y}$ & & & $\mathrm{E}$ & \\
\hline - Voronoi-based & {$[27]$} & & $\mathrm{Y}$ & & & $\bar{E}$ & \\
\hline \multicolumn{8}{|l|}{ 5. Hybrid Structure } \\
\hline \multicolumn{8}{|l|}{ Complex vehicular traffic model } \\
\hline - Free way mobility & {$[6]$} & & & & & $\mathrm{E}$ & \\
\hline - Manhattan Mobility model & {$[28,29]$} & & & & & $\mathrm{Y}$ & \\
\hline $\begin{array}{l}\text { User -Oriented mobility data- } \\
\text { model }\end{array}$ & {$[30]$} & & & $\mathrm{Y}$ & $\mathrm{Y}$ & $\mathrm{Y}$ & \\
\hline \multicolumn{8}{|l|}{ Tactical scenario Model } \\
\hline - Catastrophe-scenario & {$[32]$} & $\mathrm{Y}$ & $\mathrm{Y}$ & & & $\mathrm{Y}$ & \\
\hline - platoon & {$[33]$} & & & & $\mathrm{Y}$ & $\mathrm{Y}$ & \\
\hline Disaster-area model & [5] & $\mathrm{Y}$ & $\mathrm{Y}$ & $\mathrm{Y}$ & $\mathrm{Y}$ & $\mathrm{Y}$ & $\mathrm{Y}$ \\
\hline
\end{tabular}

Note - " $Y$ "- represents explicitly modeled and "E"- represents not modeled but could be extended to meet tactical requirements. 


\section{Conclusion}

The mobility models are categorized on account of mobility matrices are presented in table 2 . and it also analyses which mobility models are fulfilling the requirements of tactical scenario. Paper highlights that some of model strongly satisfy separate tactical requirements like geographic division based model satisfy tactical area, reference point group satisfy group movement, structure group \& community based model satisfy couple of tactical requirement optimal path and group movements, etc are tabulated in table 2. It could be possible that some of model integrated in other models to attain tactical scenario requirements together e.g. group movement can be easily integrated using reference point approach.

Beside the Disaster-area model no other model that sum up the entire tactical requirement together. The Disaster-area model which realizes the tactical requirements in details. This model is useful in performance evaluation of military operation.

\section{References}

[1] T. Camp, J. Boleng, and V. Davies, "A survey of mobility models for ad hoc network research", Wirel. Commun. Mob. Comp., vol. 2 no. 5, pp. 483-502, 2002.

[2] Babak Pazand, Chris McDonald," A Critique of Mobility Models for Wireless Network Simulation", $6^{\mathrm{TH}}$ IEEE/ACIS , ICIS 2007.

[3] F. Bai, A. Helmy, "A Survey of Mobility Modeling and Analysis in Wireless Adhoc Networks" in Wireless Ad Hoc and Sensor Networks, Kluwer Academic Publishers, 2004.

[4] Nils Aschenbruck, Elmar Gerhards-Padilla, and Peter Martini," A survey on mobility models for performance analysis in tactical mobile networks", JTIT, 2/2008, pp: 54-61.

[5] N. Aschenbruck, E. Gerhards-Padilla, M. Gerharz, M. Frank, and P. Martini, "Modelling mobility in disaster area scenarios", in Proc.10th ACM IEEE Int. Symp. Model. Anal. Simul. Wirel. Mob. Syst.MSWIM, Chania, Greece, 2007.

[6] F. Bai, N. Sadagopan, and A. Helmy, "IMPORTANT: a framework to systematically analyze the impact of mobility on performance of routing protocols for adhoc networks", in Proc. IEEE INFOCOM, San Francisco, USA, 2003, pp. 825-835.

[7] J. Broch, D. Maltz, D. Johnson, Y. Hu, and J. Jetcheva. Multi-hop wireless ad hoc network routing protocols. In Proceedings of the ACM/IEEE International Conference on Mobile Computing and Networking (MOBICOM), pages 85-97, 1998.

[8] D. B. Johnson and D. A. Maltz, "Dynamic source routing in ad hoc wireless networks", in Mobile Computing, T. Imielinski and H. Korth, Eds. Norwell: Kluwer, 1996, vol. 353, pp. 153-181.

[9] J. Yoon, M. Liu, and B. Noble, "Random waypoint considered harmful", in Proc. IEEE INFOCOM, San Francisco, USA, 2003, pp. 1312-1321.

[10] C. Bettstetter, G. Resta, and P. Santi, "The node distribution of the random waypoint mobility model for wireless ad hoc networks", IEEE Trans. Mob. Comp., vol. 2, no. 3, pp. 257-269, 2003.

[11] D. M. Blough, G. Resta, and P. Santi, "A statistical analysis of the long-run node spatial distribution in mobile ad hoc networks", Wirel.Netw., vol. 10, pp. 543-554, 2004.

[12] C. Bettstetter and C. Wagner, "The spatial node distribution of the random waypoint mobility model”, in Proc. 1st German Worksh. Mob. Ad-Hoc Netw. WMAN'02, Ulm, Germany, 2002, pp. 41-58.

[13] E. M. Royer, P. M. Melliar-Smith, and L. E. Moser, "An analysis of the optimum node density for ad hoc mobile networks", in Proc. IEEE Int. Conf. Commun., Helsinki, Finland, 2001, vol. 3,pp. 857-861.

[14] An Huiyao, Lu Xicheng, and Peng Wei” A Cluster-Based Multipath Routing for MANET", Computer School, National University of Defense Technology ,Changsha, China, pp.405-413 
[15] Dali Wei and H Anthony Chan,” An Efficient Clustering Algorithm for Topology Maintenance and Energy Saving in MANETs", Department of Electrical Engineering, University of Cape Town, May $1,2006$.

[16] B. Liang and Z. Haas. Predictive distance-based mobility management for PCS networks. In Proceedings of the Joint Conference of the IEEE Computer and Communications Societies (INFOCOM), March 1999.

[17] C. Bettstetter, "Mobility modeling in wireless networks: categorization,smooth movement, and border effects", ACM SIGMOBILE Mob. Comp. Commun. Rev., vol. 5, no. 3, pp. 55-66, 2001.

[18] C. Bettstetter, "Smooth is better than sharp: a random mobility model for simulation of wireless networks", in Proc. 4th Int. Symp.Model. Anal. Simul. Wirel. Mob. Syst. MSWIM, Rome, Italy, 2001,pp. 19-27.

[19] X. Hong, M. Gerla, G. Pei, and C.-C. Chiang, "A group mobility model for ad hoc wireless networks", in Proc. Int. Symp. Model. Simul. Wirel. Mob. Syst. MSWiM, Seattle, USA, 1999, pp. 5360 .

[20] K. Blakely and B. Lowekamp, "A structured group mobility modelfor the simulation of mobile ad hoc networks", in Int. Conf. Mob.Comp. Netw., Proc. 2nd Int. Worksh. Mob. Manag. Wirel. Acc. Pro-toc., Philadelphia, USA, 2004, pp. 111-118.

[21] M. Musolesi and C. Mascolo, "A community based mobility model for ad hoc network research", in Proc. 2nd ACM/SIGMOBILE Int.Worksh. Multi-hop Ad Hoc Netw. Theory Real. REALMAN'06, Colo-cated with MobiHoc2006, Florence, Italy, 2006, pp. 31-38.

[22] J. Tian, J. H“ahner, C. Becker, I. Stepanov, and K. Rothermel, "Graphbased mobility model for mobile ad hoc network simulation", in Proc. 35th Ann. Simul. Symp., San Diego, USA, 2002, pp. 337-344.

[23] S. Bittner, W.-U. Raffel, and M. Scholz, "The area graph-based mobility model and its impact on data dissemination", in Proc. IEEE PerCom, Kuaai Island, Hawaii, USA, 2005, pp. 268-272.

[24] M. Guenes and J. Siekermann, "CosMos - communication scenario and mobility scenario generator for mobile ad-hoc networks", in Proc. 2nd Int. Worksh. MANETs Interoper. Iss. MANETII'05, Las Vegas, USA, 2005.

[25] A. Jardosh, E. M. Belding-Royer, K. C. Almeroth, and S. Suri, “Towards realistic mobility models for mobile ad hoc networks", in Proc. IEEE MobiCom, San Diego, USA, 2003, pp. 217-229.

[26] A. P. Jardosh, E. M. Belding-Royer, A. K. C., and S. Suri, "Realworld environment models for mobile network evaluation", IEEE J. Selec. Areas Commun., vol. 23, no. 3, pp. 622-632, 2005.

[27] H.-M. Zimmermann and I. Gruber. "A Voronoi-based mobility model for urban environments", in Eur. Wirel. 2005 Conf., Zypern, Greece, 2005.

[28] Jerome Harri, Fethi Filali and Christian Bonnet" Mobility Models for Vehicular Ad Hoc Networks: A survey and taxonomy", Research Report RR-06-168, Institut Eur'ecom, Department of Mobile Communications, FRANCE, March 26, 2007.

[29] Bhavyesh Divecha, Ajith Abraham, Crina Grosan, Sugata Sanyal," Impact of Node Mobility on MANET Routing Protocols Models" Mumbai University, India, pp.1-11

[30] I. Stepanov, J. H“ahner, C. Becker, J. Tian, and K. Rothermel,"A meta-model and framework for user mobility in mobile networks",in Proc. 11th Int. Conf. Netw. ICON 2003, Sydney, Australia,2003, pp. 231-238.

[31] I. Stepanov, P. J. Marron, and K. Rothermel, "Mobility modeling of outdoor scenarios for manets", in Proc. 38th Ann. Simul. Symp. ANSS'38, San Diego, USA, 2005, pp. 312-322.

[32] P. Johansson, T. Larsson, N. Hedman, B. Mielczarek, and M. Degermark, "Scenario-based performance analysis of routing protocols for mobile ad-hoc networks", in Proc. IEEE MobiCom, Seattle, USA, 1999, pp. 195-206.

[33] S. Reidt and S. D. Wolthusen, "An evaluation of cluster head TA distribution mechanisms in tactical MANET environments”, in Proc.Conf. ACITA, College Park, USA, 2007. 


\section{Authors :}

Santosh Kumar (sanjydpt@iitr.ernet.in) received M.Tech. in Computer Science \& Engineering from Aligarh Muslim University, Aligarh (India) in 2007 \& B.E. in Information Technology from C.C.S. University, Meerut (India) in 2003, the member of ACEEE, ISOC (USA), has published over seven research papers in National \& International Journals/conferences in field of Wireless Communication Networks, Mobile Computing and Grid Computing. He has more than 3 years of experience in teaching of UG (B.Tech.) \& PG (M.Tech.) level courses in teaching as a Lecturer/Assistant Professor. Currently, he is a Doctoral candidate in Indian Institute of Technology, Roorkee (India) in the field of Wireless Communication Networks since august 2008.

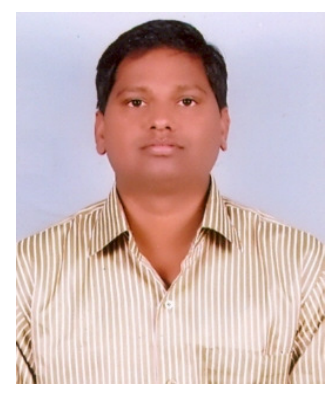

Dr. S.C. Sharma (scs60fpt@iitr.ernet.in) received M.Tech. (Electronics \& Communication Engg. and Ph.D. Electronics \& Computer Engg.) in 1983 and 1992 respectively from IIT Roorkee (erstwhile University of Roorkee), the honorary member of NSBE, ISOC, and IAENG, ACEEE. He started his career as R \& D Engineer in 1983 then joined teaching profession in Jan. 1984 in IITRoorkee and continuing till date. He has published over hundred thirty six research papers in national and international journals/conferences and supervised more than 30 projects/dissertation of PG students. He has supervised several Ph.D. in the area of Computer Networking, Wireless Network, Computer Communication and continuing supervising Ph.D. in the same area.

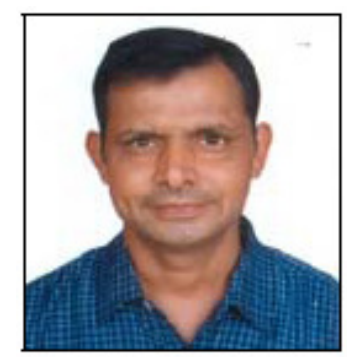
Currently, he is supervising Six Ph.D. Scholars. He has successfully completed several major research projects independently and many minor research projects related to Communication and SAW filter Design sponsored by Government of India. IIT-Roorkee has awarded him the Khosla annual research prize with best research paper in the year 2000. His many research papers have been awarded by National and International Committees. He has worked as research scientist at FMH, Munchen, Germany in the year 1999. He is the active reviewer of IEEE Sensor Journal and Chief Editor of two reputed International Journals and Editor of National Journal (BITS, PILANI). He has also worked as Group leader of Electronics \& Instrumentation Engg. Department of BITS-Pilani-Dubai Campus, from Aug. 2003 to Aug. 2005. Presently he is continuing as Associate Professor at IIT Roorkee.

BhupendraSuman (bhupendra.suman@rediffmail.com) received his B.E (Electronics \& Communication Engineering) from MANIT Bhopal, India in

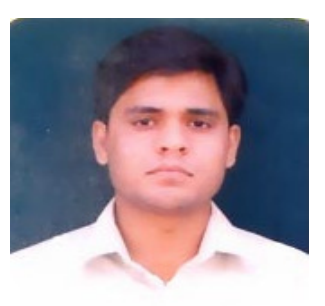
2000 and joined DRDO in Defence Electronics Application Laboratory(DEAL)

Dehradun. In DEAL he has worked in various communication projects including Combat Net Radio (CNR). He was awarded Laboratory Scientist of the year 2005 for his contribution in project. Presently he is working in the field of tactical MANET as a Doctoral candidate at IIT Roorkee since January 2009. 